\title{
The VLBI digital terminal at the NASA's Deep Space Network: first results from non-JPL correlators
}

\section{García-Miró}

Madrid Deep Space Communications Complex/NASA, INTA/ISDEFE, Spain

E-mail: cgmiro@mdscc.nasa.gov

\section{J.E. Clark, C.S. Jacobs, S.P. Rogstad}

Jet Propulsion Laboratory, California Institute of Technology/NASA, USA

\section{R.M. Campbell, M. Kettenis}

Joint Institute for VLBI in Europe, the Netherlands

\section{S. Horiuchi}

Canberra Deep Space Communications Complex/NASA, CSIRO, Australia

\section{L.G. Snedeker}

Goldstone Deep Space Communications Complex/NASA, SaiTech, USA

The NASA's Deep Space Network (DSN) has replaced the Mark IV Data Acquisition Terminal (DAT) with a digital backend, the DSN VLBI Processor (DVP). The DVP is an in-house JPL development that uses a CASPER ROACH board for real-time digital signal processing and channelization and streams the data into a Mark-5C recorder in VDIF format. The digital terminal maximum data rate of $2 \mathrm{Gbps}$ is a considerable improvement over the previous generation. Future upgrades may enable 4 Gbps data rates.

The DVP has been successfully supporting JPL VLBI Astrometry and Earth Orientation observations since 2013, using the JPL software correlator. As the DVP does not use the standard Field System environment to perform the VLBI observations, efforts are under way to make it compatible with non-JPL correlators by providing the required monitor and calibration data in the appropriate format. This paper documents the first successful results obtained with the DVP and the European VLBI Network (EVN), using the EVN software correlator at the Joint Institute for VLBI in Europe (JIVE).

Acknowledgements: Research done in part under NASA contract. Sponsorship by U.S. Government, and our respective institutes acknowledged. Copyright (C)2015. All Rights Reserved.

12th European VLBI Network Symposium and Users Meeting,

7-10 October 2014

Cagliari, Italy 


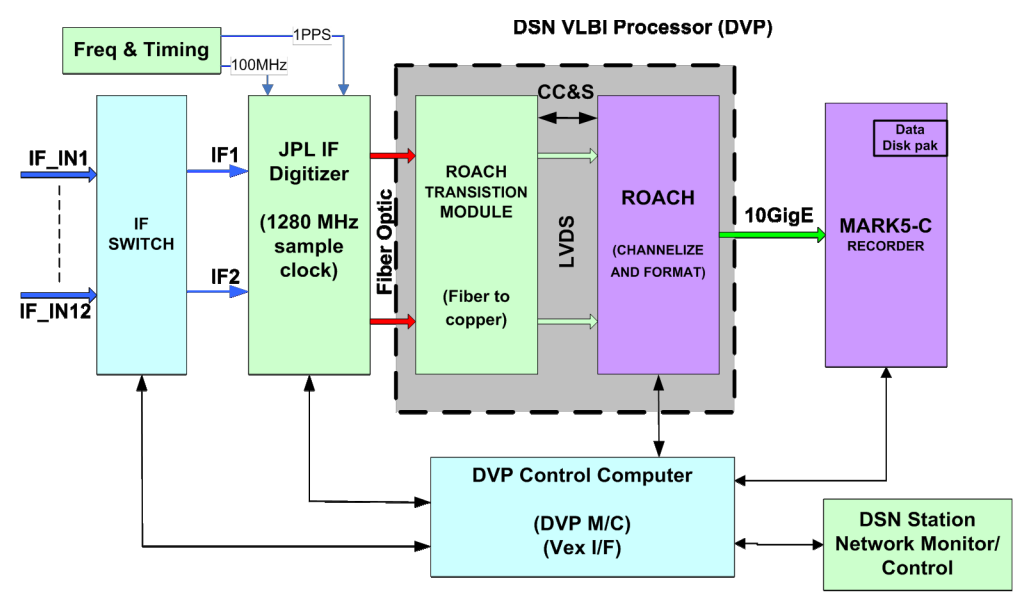

Figure 1: DVP block diagram with its different components: IF switch, digitizer, ROACH board with transition module, Mark-5C recorder and Data Processor and Controller computer.

\section{Introduction}

The NASA's Deep Space Network is the largest and most sensitive scientific communications system in the world primarily designed to support interplanetary spacecraft missions, but it also performs radar and radio astronomy observations. It realizes and maintains an extragalactic reference frame for JPL navigation purposes (García-Miró et al., these proceedings [2]) and participates in the observations of the European VLBI Network, the International VLBI Service for Geodesy and Astrometry, and the Australian Long Baseline Array. During 2014 the DSN has been celebrating 50 years of communication and discoveries.

Recently the DSN has replaced the MarkIV DAT hardware with a modern digital backend system, the DSN VLBI Processor (DVP). The DVP has been successfully supporting JPL VLBI Astrometry and Earth Orientation observations since 2013, using the JPL software correlator. Efforts to validate its proper function and interoperability in international VLBI networks are in progress and have already obtained positive results with most of them, including DiFX-based correlators (Bertarini \& Philips, priv. comm, 2014). This contribution focuses on the first successful DVP fringes that were obtained outside the DSN with the EVN network and the EVN software correlator at JIVE (SFXC), (Keimpema et al. [4], Kettenis et al., these proceedings [5]).

\section{DSN VLBI Processor Overview}

The DVP, the new DSN VLBI Processor (Navarro et al., 2011 [7]; García-Miró et al., 2012 [3]), is a JPL development partially compatible with other digital backends (DBBC, RDBE, etc.). Fig. 1 shows its different components.

Unlike most VLBI sites, each DSN complex has many antennas available for VLBI observations each with different receiving bands. An IF switch selects from amongst 12 analog inputs at the DSN intermediate band (IF) of approximately 100-600 MHz, in order to support several bands from at least 3 antennas per complex. The switch selects two analog IF outputs that are digitized 
using the JPL IF sampler module, providing A/D 8-bit samples at $1280 \mathrm{MHz}$ for $640 \mathrm{MHz}$ of digitized bandwidth. However, there a guard bands on either end that make 0-100 MHz and 620-640 $\mathrm{MHz}$ impractical to use.

Digital Signal Processing and Channelization is performed using a ROACH board (Casper, 2012 [1]) in two stages: a polyphase filter bank breaks up the input signal into 7 fixed bands of data, each $160 \mathrm{MHz}$ wide, and a second stage that selects one of the seven wideband inputs, applies a digital mixer for precise channel location selection and uses a cascade of downconverting filters (Cascaded Integrator-Comb -CIC-\& Finite Impulse Response -FIR- filters). The DVP records up to 32 upper/lower or 16 complex sub-channels. The output bandwidth per channel is variable from $16 \mathrm{MHz}$ to $0.5 \mathrm{KHz}$ (or from $32 \mathrm{MHz}$ to $1 \mathrm{KHz}$ for the complex sub-channels) with currently only 2 bits available per sub-channel.

The DVP streams the data from the ROACH board through a 10GigE connection to a Mark5C recorder (Haystack, 2008 [6]) providing up to 2 Gbps data rates with the goal of achieving 4 Gbps. Data is stored on Mark-5 disk packs in VDIF format (16 bytes legacy header and single data threads carrying multi-channel data frames).

The PC-Field System computer has been replaced by the Data Processor and Controller (DPC) computer that uses a custom-made application to configure and run the observations using VEX 1.5 schedules from the users. The standard Field System application is not used therefore efforts are under way to maintain compatibility with other VLBI centers. The DPC computer interfaces to the DSN Monitor \& Control infrastructure (NMC), which interacts with all DSN subsystems via directives, responses and monitor data, using the DSN automation scheme. The phase calibration signal is extracted in real-time, with feedback at the DVP graphical user interface.

\section{First DVP fringes within the EVN network}

DVP fringes have been demonstrated for first time outside the DSN for a 1 Gbps L-band observation performed by Robledo's 70m antenna during the EVN observing session III, 2013. Note that only L-LCP is available at Robledo and both the DVP and the MarkIV DAT were used. JIVE adapted the SFXC software correlator to process the particular DSN VDIF format.

Comparison between the DVP (Rd) and the MarkIV DAT (Ro) results shows better performance for the digital backend. Fringes are stronger with the DVP for the same scan (Fig.2) as the digital filters have less instrumental artifacts, with a linear phase response and no channel-tochannel variations in the filters (Fig. 3).

\section{Conclusions}

The DSN digital backend (DVP) has enhanced considerably the quality of the DSN VLBI observations. Among many other advantages, the JPL digitizer module decreases the spurious signals and the digital linear filters have reduced instrumental artifacts and eliminated channel-tochannel variations. The DVP has now been validated within the EVN network. Comparison with the MarkIV DAT shows its better performance. The usage of the Mark-5C recorder has allowed us to increase the recording rate up to a maximum of 2,048 Mbps thus sampling $512 \mathrm{MHz}$ of available instantaneous analog bandwidth which provides unprecedented sensitivity for the DSN. 
Amplitude for EP087E_DVPtstLAG

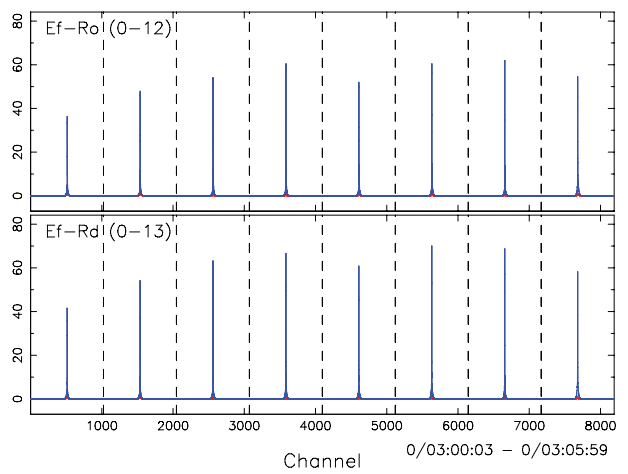

Amplitude for EP087E_DVPtst (LCP only)

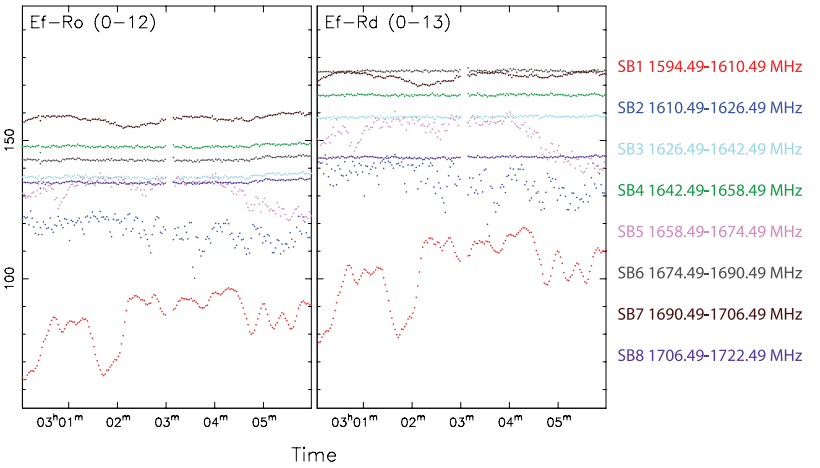

Figure 2: Left. Lag-based fringes between Effelsberg and Robledo MarkIV DAT (Ro) and Robledo DVP (Rd) for all the frequency channels ( 8 channels or sub-bands, $16 \mathrm{MHz}$ BW, 2 bits, blue is LL) averaged for the first two scans. Note that fringes are about 15\% stronger with the DVP than with the MarkIV DAT as a result of the better performance of the digital filters (Fig. 3). Right. Plot of the raw correlation amplitude (milli-amp) versus time for every frequency channel for Robledo-Effelsberg baseline for the same timerange, averaged in frequency across the middle $80 \%$ of the band. For the same frequency channel the baseline amplitude is stronger for Rd, with remarkable difference for e.g. the sub-band SB6 (in grey) again due to non-flat variation of phase versus frequency for Ro (Fig. 3). Note that scatter in some channels is caused by L-band RFI, mostly in sub-bands SB1 (in red) and SB2 (in blue).

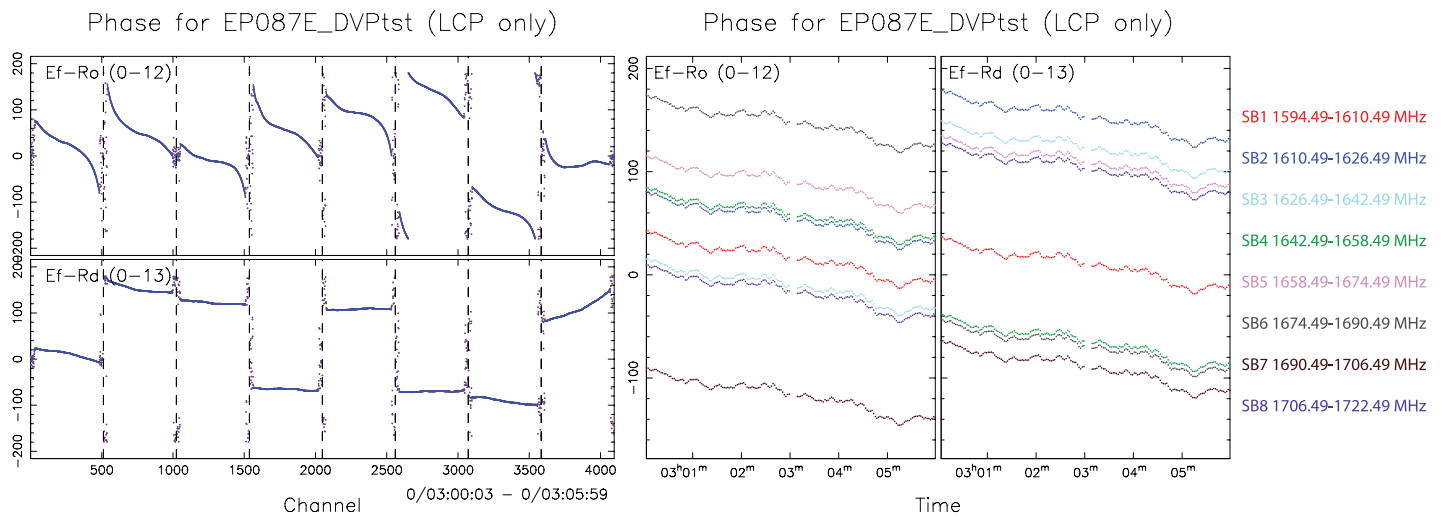

Figure 3: Left. Plot of the baseline phase (deg) versus frequency for every channel. for Robledo-Effelsberg baseline averaged for the first two scans. Better performance is found from the digital filters (Rd) than from the analog ones (Ro) which have flatter phase vs. frequency variation. Right. Plot of the baseline phase (deg) versus time for each channel, averaged in frequency across the middle $80 \%$ of the band. Phase variations are similar for both terminals with a constant phase offset per channel.

\section{References}

[1] CASPER, Reconfigurable Open Architecture Computing Hardware (ROACH), 2012.

[2] C. García-Miró, et al., The X/Ka Celestial Reference Frame, in proc. of 12th EVN Symposium, 2015.

[3] C. García-Miró, et al., VLBI DAT modernization at the DSN, in proc. of IVS GM, 2012.

[4] A. Keimpema, et al., The SFXC software correlator, submitted to Experimental Astronomy.

[5] M. Kettenis, A. Keimpema, SFXC, in proc. of 12th EVN Symposium, 2015.

[6] MIT Haystack Observatory, Mark 5C Specification, Mark5 memo \#57, 2008.

[7] R. Navarro, et al., Impl. Issues and Choices for VLBI DAT in DSN, 9th US VLBI Tech. M., 2011. 